\title{
A bicontinuous cubic phase of monoolein and water as medium for electrophoresis of both membrane-bound probes and DNA
}

\author{
Nils Carlsson*, Nima Sanandaji*, Marina Voinova ${ }^{\#}$ and Björn Åkerman*†
}

\section{Supplementary information.}

Diffusion of $\left[\mathrm{Ru}(\mathrm{phen})_{2} \mathrm{Me}_{2} \mathrm{dppz}\right]^{2+}$ into the cubic phase as a function of time (Figure $\mathrm{S} 1$ ). At each given time (min) a pair of cuvettes are shown. The right-hand cuvette contains a sample of the cubic phase in its bottom third, the left-hand one is the monoolein-free control. At 0 minutes the two cuvettes contain water only. A $100 \mu \mathrm{M}$ aqueous solution of $\left[\mathrm{Ru}(\mathrm{phen})_{2} \mathrm{Me}_{2} \mathrm{dppz}\right]^{2+}$ in $\mathrm{pH} 5$ acetate buffer was then added to both cuvetttes, and the pair was scanned at indicated times ( $\mathrm{min})$.

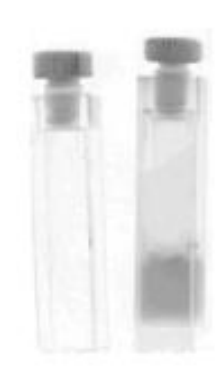

0 min

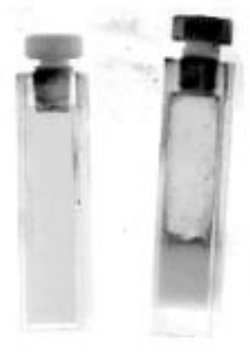

3

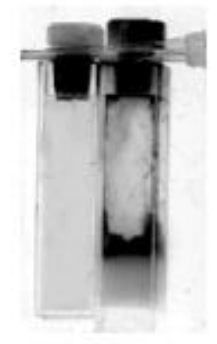

1030

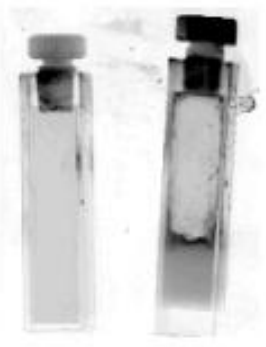

9

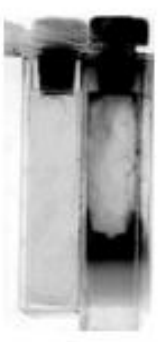

2618

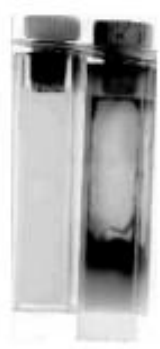

4264

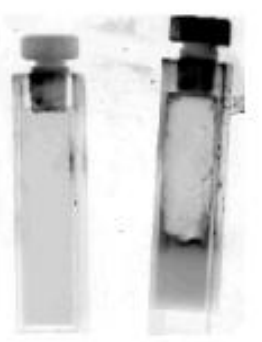

21
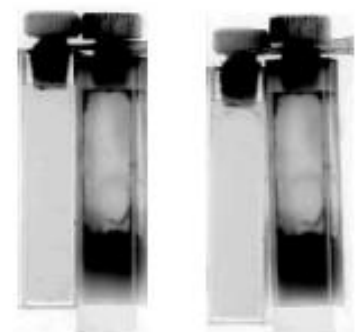

9824

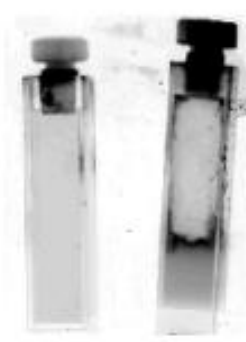

71

Figure S1. 
The almost undetectable increase in fluorescence in the left-hand cuvette between 0 and 3 minutes show the weak fluorescence of $\left[\mathrm{Ru}(\mathrm{phen})_{2} \mathrm{Me}_{2} \mathrm{dppz}\right]^{2+}$ in water. The progressing front in the right-hand cuvette shows that the dye diffuses into the cubic phase, and the strongly enhanced fluorescence intensity compared to the control supports it is inserted in the monoolein membrane (Ardhammar, M. Lincoln,P., Nordén. B 2001. J. Phys. Chem. B. 105. 11363-11368.) The time-evolution of the position of the front was used to evaluate a diffusion coefficient (see text).

Theoretical distribution of pore-radius of the aqueous channels in the diamond-type cubic phase of monoolein. (Figure S2). Derived from the diamond minimal surface (lattice parameter $109 \AA$ ), after decorating it with a $34 \mathrm{~nm}$ thick lipid bilayer. The distribution is bimodal, with approximately $75 \%$ of the pore area belonging to the major peak at larger radii. The average pore radius is $55 \AA$.

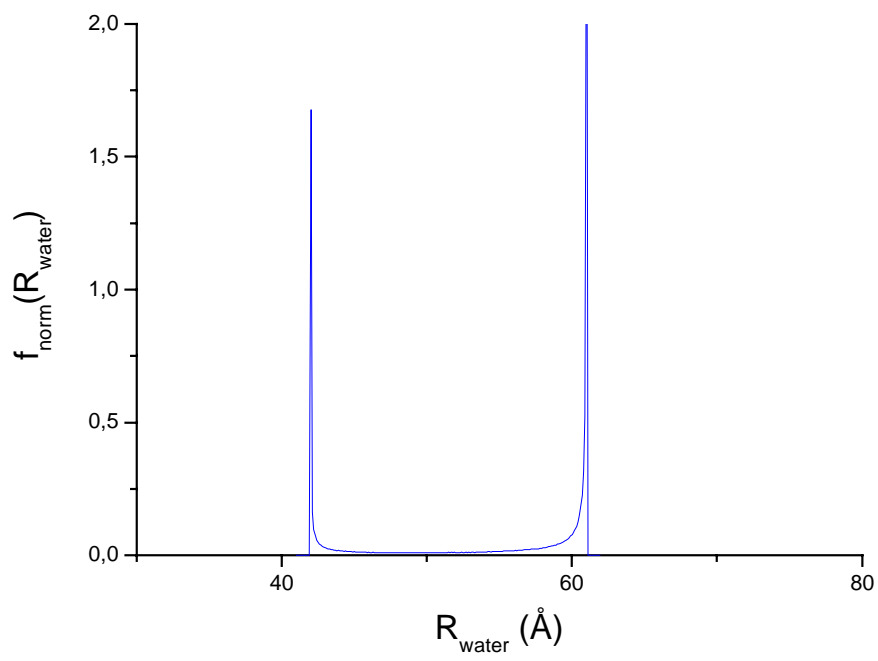

Figure S2. 